\title{
The Conundrum of the Efficacy of Tribulus terrestris Extract as Supplement for Muscle Mass Gain
}

\author{
Renato M Salgado ${ }^{1^{*}}$, Celso F Carvalho ${ }^{2}$, Carlos V Andreoli ${ }^{3}$ and Aléssio C Mathias ${ }^{1^{*} \#}$ \\ ${ }^{1}$ Genesis Clinic - São Paulo, Brazil \\ ${ }^{2}$ Plenitas Clinic - São Paulo, Brazil \\ ${ }^{3}$ Department of Orthopaedics and Traumatology, UNIFESP - São Paulo, Brazil
}

*Corresponding author: Renato M Salgado, Genesis Clinic - São Paulo, Brazil, Tel: +1 (979) 985-7625; E-mail: rmsalgado81@gmail.com

Aléssio C Mathias, Genesis Clinic - São Paulo, Brazil, E-mail: alessio@clinicagenesis.com.br

Received date: Dec 06, 2017; Accepted date: Dec 21, 2017; Published date: Dec 27, 2017

Copyright: @ 2017 Salgado RM, et al. This is an open-access article distributed under the terms of the Creative Commons Attribution License, which permits unrestricted use, distribution and reproduction in any medium, provided the original author and source are credited.

\#These authors have equally contributed to this paper.

\begin{abstract}
The dried extract of Tribulus terrestris has been widely applied in traditional medicine as alternative treatment for many ailments. Its main active components are steroidal saponins, which are thought to induce the reduction of testosterone into the potent dihydrotestosterone, thus possibly enhancing steroid hormone-related characteristics. In fact, these herbs are still used to treat some sexual dysfunctions and as male sexual stimulant. It is well-known that many individuals attempt to gain muscle mass by the intake of natural or synthetic anabolic products, including dietary supplements containing Tribulus. In this light, pharmaceutical advances have promoted a conspicuous increase in the commercialization of such products with alleged androgenic properties. Several studies with $T$. terrestris supplementation involving domestic and laboratory animals, as well as human subjects, have shown conflicting data with regard to increase in male sex hormone levels, sperm quality and physical strength, among other attributes. The aim of this review was to investigate the existing evidence on the impact of $T$. terrestris supplementation on lean body mass, and to address its reliability. Different database sources and sets of key words were used to search the related literature. Considering a set of predetermined inclusion criteria, the contents of three studies were explored. In these studies there were no significant differences in body composition parameters, such as lean mass and body fat, between control (placebo) and experimental groups. However, the main reason for caution in these reports was the overall reduced sample size, which precludes a firm conclusion from the obtained results. Further investigation by conducting robust randomized controlled trials is still warranted.
\end{abstract}

Keywords: Tribulus terrestris, Saponin; Testosterone; Lean body mass; Strength; Muscle gain; Review

\section{Introduction}

It is notorious that many individuals, especially male athletes and bodybuilders, constantly seek a boost in steroid hormone levels by the intake of natural or synthetic anabolic products, with the goal of improving the performance in their respective sport, and their physical appearance. Pharmaceutical advances have allowed the production of relatively potent androgenic drugs with fewer side effects; indeed the thriving dietary supplement market offers a wide variety of natural products with supposedly positive effects on athletic performance and lean mass gain $[1,2]$.

There are a variety of plants thought to improve male fertility, gain of lean muscle mass, and decrease of body fat percentage, among other attributes [3]. Among these well-known plants are the dicotyledonous of the genus Tribulus of the Zygophyllaceae family, which comprise about 20 species of shrubs or herbs that grow mainly in subtropical areas around the world [4]. These herbs have been used as tonic, palliative, astringent, stomachic, antihypertensive, diuretic, and urinary disinfectant, as well as male sexual stimulant and an alternative treatment for sexual dysfunctions [5,6]. Tribulus terrestris is the most common species among them and has been used as traditional medicine for thousands of years [7]. Considering its various phytochemical components, majorly present are furostanol glycosides or saponins, such as protodioscin and protogracillin, of which protodioscin is commonly the most abundant [8]. It is suggested that a major role of this steroidal saponin is to induce the reduction of testosterone into the potent dihydrotestosterone (DHT), which occurs by the action of the enzyme 5 -alpha reductase.

A number of reports with animal models have been published upto-date, concerning the effects of supplementation with Tribulus extracts. Gauthaman et al. [9] performed a prospective randomized study in which male rats were treated for 8 weeks with different oral doses of T. terrestris extract: $2.5,5$ and $10 \mathrm{mg} / \mathrm{kg}$ body weight, and subjected to sexual behavior studies. The authors reported a significant increase in body weight $(9,23$, and $18 \%$, respectively), and suggested that weight gain and improvement in sexual behavior parameters may be related to the androgen enhancing properties of T. terrestris. Later, Gauthaman and Ganesan [10] showed that treatment of rats, rabbits and rhesus monkeys with T. terrestris induced an increase in the serum concentration of two male sex hormones, testosterone and DHT. In boars, supplementation with $T$. terrestris increased the levels of dehydroepiandrosterone (DHEA), LH and testosterone; in addition, it stimulated the conversion of testosterone into DHT [11].

More recently, Yin et al. [12] demonstrated that lean mass gain and serum testosterone levels increased significantly in male rats submitted to overtraining after three to four weeks of $T$. terrestris treatment 
Page 2 of 4

administered intragastrically. In contrast, a previous study showed that consumption of T. terrestris dry extract (dissolved in the water) by male rats during 28 days did not change serum testosterone levels, regardless of the doses given (11, 42 and $110 \mathrm{mg} / \mathrm{kg} /$ day) [13]. Several studies have reported enhancement in semen parameters and sexual performance in different species; for simplicity in the present review, only studies using an animal model that evaluated alterations in sex hormone levels or body composition were included. A recent review by Neychev and Mitev [14] suggests that there is evidence showing beneficial effects of $T$. terrestris on libido and erectile dysfunction in humans and other animals, but that it is inconclusive whether these effects are directly related to androgen-enhancing properties.

Brown and co-workers [15], in a prospective study with adult men (age range: 30-59 years old), showed that the intake of an oral preparation containing androstenedione, DHEA, saw palmetto, indole-3-carbinol, chrysin, and $T$. terrestris during 28 days, significantly increases serum levels of androstenedione (342\%), free testosterone (38\%) and DHT (71\%). In a recent randomized controlled trial, overweight and obese men were given either $500 \mathrm{mg}$ of placebo or $500 \mathrm{mg}$ of a preparation containing T. terrestris and three other herb extracts; overall fat percentage was significantly decreased in the experimental group after a 6-month period [16]. Nevertheless, studies in humans commonly present the use of combined supplements or drugs by the subjects in the experimental group, which in turn precludes a firm conclusion on the individual effectiveness of these products. A major concern is the recurrent use of commerciallyavailable T. terrestris supplements, with unknown concentration of saponins and possible contamination of the preparation [17].

The work of Roaiah et al. [18] demonstrated a significant increase in free testosterone levels and a positive impact on erectile function after three months of treatment with $T$. terrestris extract by male patients suffering from partial androgen deficiency. A recent study by Salgado and co-workers [3] in a clinical human IVF setting demonstrated that treatment with $T$. terrestris can improve sperm concentration and motility in the ejaculate of men with reduced sperm quality. These results partially corroborate those of a previous clinical trial in oligozoospermic men undergoing infertility treatment in which a significant increase in sperm concentration, but not motility, was reported after oral administration of $T$. terrestris, pregnancies were achieved for 8/36 men in the T. terrestris treatment group, vs. 0/9 in the control group [19]. It has been hypothesized that T. terrestris is able to induce augment in sperm concentration in the ejaculate by increasing endogenous testosterone levels, thus improving spermatogenesis in the seminiferous tubules. Theoretically, this event should occur via a prior increase in GnRH levels, which in turn stimulates the production of LH and FSH [20].

The relationship between $T$. terrestris supplementation and steroid hormone levels or attributes related to sexual performance has been assessed in many original and review articles, with controversial data and opinions. However, peer-reviewed prospective studies on the efficacy of $T$. terrestris as an androgenic supplement, capable of stimulating lean muscle mass gain in men and women, are still scarce. The aim of the present review was to summarize the existing evidence on the alleged impact of $T$. terrestris supplementation on lean body mass, and to address its reliability.

\section{Materials and Methods}

This review was written inspired on guidelines by Pautasso [21].

\section{Data sources}

A total of four Databases were sought: Pubmed, ScienceDirect, Web of Knowledge and Sage Journals Online. Combination of two of the following key words was used in each database: "Tribulus" and strength or skeletal muscle or lean mass or muscle gain. There was no preliminary restriction to the year of the publication.

\section{Inclusion criteria}

An article was included if met all of the following characteristics:

- Randomized placebo-controlled trial.

- Male subjects without significant differences in baseline parameters between control and experimental groups before the onset of the study.

- Oral administration of only $T$. terrestris extract by the experimental group.

- Analysis and comparison between groups, in the beginning and in the end of the study, of at least one of the following parameters: total body mass, lean muscle mass, percentage of body fat.

- Supervised physical activity routine.

- Article published in English.

\section{Results and Discussion}

After the selection process, three articles were analyzed in this review. Table 1 presents the reference of the articles included, the sample size for each group, the baseline parameters, and the $T$. terrestris preparation details; Table 2 presents the subject type, the duration and the main results of each study (related to the scope of this review and according to the authors' scrutiny). In the three studies there were no significant changes in the key outcome (refer to Table 2 for details) between the control group (placebo) and the group subjected to supplementation with $T$. terrestris. Rogerson et al. [22] noted a significant increase in lean mass within group (both placebo and T. terrestris), when comparing the beginning and the end of the 5week trial. Correspondingly, Salgado et al. [3] showed a significant increase in lean tissue in a group of 65 infertile men after treatment with $750 \mathrm{mg} /$ day of $T$. terrestris extract, when compared with the values at the beginning of the study; however, in that study [3], there was no placebo group as control and only intragroup variations were evaluated.

Furthermore, besides the key outcome presented in Table 2, other parameters were evaluated in those studies. In the work of Rogerson et al. [22] the strength of the athletes was measured in the beginning and in the end of the experiment, by evaluating the weight (in kilograms) lifted during the execution of the following exercises: bench press, leg press, deadlift and seated row. There was a significant increase within group along treatment, but not between groups. This is probably due to the controlled dietary intake and high intensity training plan performed by all athletes enrolled in the study. The urinary testosterone/epitestosterone (T/E) ratio was also measured in weeks 0 and 6 , and no differences were found between or within group. This test is used to detect abuse of substances with androgenic properties prior to professional competitions [23]. The use of doping agents by professional athletes is strictly prohibited and such awareness is of utmost importance [17]. 


\begin{tabular}{|c|c|c|c|c|c|c|}
\hline \multirow{2}{*}{ Article (citation) } & \multirow[t]{2}{*}{ Group } & \multirow[t]{2}{*}{$\mathbf{n}$} & \multicolumn{3}{|c|}{ Baseline parameters } & \multirow[t]{2}{*}{$\begin{array}{l}\text { Tribulus terrestris dosage } \\
\text { (d) }\end{array}$} \\
\hline & & & $\begin{array}{l}\text { Age } \\
\text { (y) }\end{array}$ & $\begin{array}{l}\text { Height } \\
(\mathrm{cm})\end{array}$ & Weight (kg) & \\
\hline $\begin{array}{l}\text { Antonio } \\
\text { et al., } 2000 \text { [20] }\end{array}$ & $\begin{array}{l}C \\
E\end{array}$ & $\begin{array}{l}7 \\
8\end{array}$ & $\begin{array}{l}22.4 \pm 3.9 \\
20.0 \pm 1.7\end{array}$ & $\begin{array}{l}177.3 \pm 3.9 \\
182.9 \pm 4.8\end{array}$ & $\begin{array}{l}81.1 \pm 9.0 \\
75.2 \pm 6.6\end{array}$ & 250 mg: 45\% spn \\
\hline $\begin{array}{l}\text { Rogerson } \\
\text { et al., 2007 [22] }\end{array}$ & $\begin{array}{l}C \\
E\end{array}$ & $\begin{array}{l}11 \\
11\end{array}$ & $\begin{array}{l}19.0 \pm 1.3 \\
20.5 \pm 3.8\end{array}$ & $\begin{array}{l}180.7 \pm 5.0 \\
182.5 \pm 3.5\end{array}$ & $\begin{array}{l}87.6 \pm 9.0 \\
88.5 \pm 10.5\end{array}$ & $450 \mathrm{mg}: 60 \% \mathrm{spn}$ \\
\hline $\begin{array}{l}\text { Ma } \\
\text { et al., } 2015 \text { [24] }\end{array}$ & $\begin{array}{l}C \\
E\end{array}$ & $\begin{array}{l}7 \\
6\end{array}$ & $\begin{array}{l}16.6 \pm 1.9 \\
16.1 \pm 1.8\end{array}$ & $\begin{array}{l}172.7 \pm 4.0 \\
174.0 \pm 8.1\end{array}$ & $\begin{array}{l}64.1 \pm 6.6 \\
62.8 \pm 15.2\end{array}$ & $1,250 \mathrm{mg}: 40 \% \mathrm{spn}$ \\
\hline
\end{tabular}

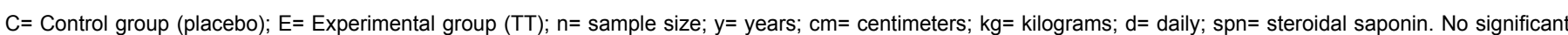
differences were observed in the baseline parameters between the two groups, in any of the studies (values of $P<0.05$ were considered statistically significant in all studies).

Table 1: Primary characteristics and treatment of the studies analyzed.

\begin{tabular}{|l|l|l|l|}
\hline Article (citation) & Subject type (male) & $\begin{array}{l}\text { Duration } \\
\text { (weeks) }\end{array}$ & Key outcome \\
\hline Antonio et al., 2000 [20] & Recreational bodybuilders & 8 & $\begin{array}{l}\text { No significant differences in percentage of body fat between or within groups } \\
(P>0.05) .\end{array}$ \\
\hline Rogerson et al., 2007 [22] & $\begin{array}{l}\text { Rugby } \\
\text { players }\end{array}$ & 5 & $\begin{array}{l}\text { No differences in body mass and lean mass between groups ( } P>0.05) . \\
\text { Increased lean mass within each group when comparing the beginning and the } \\
(P=0.07) .\end{array}$ \\
\hline Ma et al., 2015 [24] & Professional boxers & 10 & $\begin{array}{l}\text { No significant changes in total muscle mass, total fat mass, and the } \\
\text { percentages of total muscle and fat, between groups }(P>0.05) .\end{array}$ \\
\hline
\end{tabular}

Table 2: Summary of the design and main results of the studies analyzed.

Notably, one strong methodological aspect of the work of Antonio et al. [20] was the analysis of total calories and macros (carbohydrate, protein and fat) intake by the subjects along the study; no differences were observed between groups. Besides the percentage of body fat (key outcome), other features of body composition were evaluated: total body water and skinfolds. There were no significant differences between and within group. The authors suggest that the lack of improvement in overall body composition may be due to the fact that the subjects were already lean before the onset of the experiment $(\leq$ $12 \%$ body fat). This study also compared differences in the strength of the subjects before the onset and in the end of the study, and observed an increase in maximal repetitions for the leg press exercise in both control and experimental groups; curiously, only the placebo group presented a significant increase in maximal repetitions for the bench press.

The experimental design in the study of Ma et al. [24] was divided into three weeks of high-intensity training, a four-week rest period and 3 weeks of high-volume training, with continuous $T$. terrestris supplementation by the athletes. As in the other two reports, potential improvement in physical activity was evaluated; in this study the group treated with $T$. terrestris extract presented a significant increase in anaerobic performance in the end of the trial. The standard anaerobic capacity is the total amount of energy generated from the cellular anaerobic energy systems, and can be evaluated during short duration high intensity exercises [25]. Concerning the athletes' hormonal status along the study, the levels of insulin-growth factor 1 (IGF-1) and insulin growth factor binding protein 3 (IGFBP-3) were measured. Although there were no changes in plasma IGF-1 levels, a significant decrease in plasma IGFBP-3 was observed after the two training periods; IGF-1 is known to exert potential roles on muscle hypertrophy and repair [26].

Moreover, Ma et al. [24] showed no significant changes in plasma testosterone and DHT levels between and within group, along the 10week period of the study. Contrarily, Salgado et al. [3] observed a significant increase in serum DHT levels; however, there were no changes in serum DHEA and testosterone levels, after treatment with T. terrestris. In a previous study, Adimoelja and Adaikan [27] demonstrated that daily administration of $750 \mathrm{mg}$ T. terrestris extract, for a period of 3 weeks, significantly increased serum DHEA concentrations, in diabetic and non-diabetic men. These data combined indicate that the existing evidence in the literature is still controversial and requires further investigation. The potential role of $T$. terrestris in promoting the endogenous production of steroid hormones is of great interest, due to the subsequent expected effects on muscle hypertrophy and lean mass gain.

The small sample sizes, which varied from only 6 to 11 subjects per group, were the apparent weakness and the main reason for caution in the studies selected for this review. Other limitations that we may imply are the wide variation in T. terrestris dosage ( $250 \mathrm{mg}$ to 1,250 
mg of T. terrestris extract) and short duration of the experiments (5 to 10 weeks). It is important to emphasize that the purity of the $T$. terrestris extract and amount of steroidal saponins (\%) should be assessed and optimized before the onset of the study. Ideally, a much larger number of subjects should be recruited and randomly assigned to either control or experimental group in future prospective trials. Following a predefined set of criteria, such as careful diet control and training plan, is crucial to validate the outcome of these studies [2].

\section{Conclusion}

The efficacy of supplementation with T. terrestris in combination with a healthy diet and routine physical activity, with the purpose of gaining muscle mass and losing body fat, is still unclear. Further robust randomized controlled trials with strict inclusion criteria, larger sample sizes and longer periods of duration are warranted.

\section{Acknowledgement}

The authors would like to thank Mrs. Vera Lúcia Tambeiro for her invaluable support.

\section{Conflict of Interest}

The authors declare no conflict of interest.

\section{References}

1. Maughan RJ, Greenhaff PL, Hespel P (2011) Dietary supplements for athletes: emerging trends and recurring themes. J Sport Sci 29: 57-66.

2. Ahmed Qureshi, Declan Naughton, Andrea Petroczi (2014) A Systematic Review on the Herbal Extract Tribulus terrestris and the Roots of its Putative Aphrodisiac and Performance Enhancing Effect. J Diet Suppl 11: 64-79.

3. Salgado RM, Marques-Silva MH, Gonçalves E, Mathias AC, Aguiar JG, et al. (2017) Effect of oral administration of Tribulus terrestris extract on semen quality and body fat index of infertile men. Andrologia 49: e12655.

4. Hegnauer R (1973) Chemataxonomie der Pflanzen. Basel, BirkhäuserVerlag 6: 707.

5. El-Tantawy WH, Temraz A, El-Gindi OD (2007) Free serum testosterone level in male rats treated with Tribulus alatus extracts. Int Braz J Urol 33: 554-558.

6. Chhatre S, Nesari T, Somani G, Kanchan D, Sathaye S (2014) Phytopharmacological overview of Tribulus terrestris. Pharmacogn Rev 8: 45-51.

7. Melnyk JP, Marcone MF (2011) Aphrodisiacs from plant and animal sources-A review of current scientific literature. Food Res Int 44: 840-850.

8. Kostova I, Dinchev D (2005) Saponins in Tribulus terrestris- chemistry and bioactivity. Phytochem Rev 4: 111-137.

9. Gauthaman K, Ganesan AP, Prasad RN (2003) Sexual effects of puncturevine (Tribulus terrestris) extract (protodioscin): an evaluation using a rat model. J Altern Complement Med 9: 257-265.

10. Gauthaman K, Ganesan AP (2008) The hormonal effects of Tribulus terrestris and its role in the management of male erectile dysfunction an evaluation using primates, rabbit and rat. Phytomedicine 15: 44-54.
11. Opletal L, Rozkot M, Lustyková A, Frydrychová S, Daněk P, et al. (2008) Natural compounds potentially influencing pig reproduction - review. Res Pig Breed 2: 50-54.

12. Yin L, Wang Q, Wang X, Song LN (2016) Effects of Tribulus terrestris saponins on exercise performance in overtraining rats and the underlying mechanisms. Can J Physiol Pharmacol 94: 1193-1201.

13. Martino-Andrade AJ, Morais RN, Spercoski KM, Rossi SC, Vechi MF, et al. (2010) Effects of Tribulus terrestris on endocrine sensitive organs in male and female Wistar rats. J Ethnopharmacol 127: 165-170.

14. Neychev V, Mitev V (2016) Pro-sexual and androgen enhancing effects of Tribulus Terrestris L.: Fact or Fiction. J Ethnophamarcol 179: 345-355.

15. Brown GA, Vukovich MD, Martini ER, Kohut ML, Franke WD, et al. (2001) Effects of androstenedione-herbal supplementation on serum sex hormone concentrations in 30- to 59-year-old men. Int J Vitam Nutr Res 71: 293-301.

16. Vijayakumar TM, Sravanthia A, Vinodhini VM, Kumar RCS (2017) A randomized, double-blind, placebo controlled trail of Oberay (Ayurvedic Proprietary Medicine) in overweight and obese adult population. Obesity Medicine 8: 6-12.

17. RJ Maughan (2005) Contamination of dietary supplements and positive drug tests in sport. J Sports Sci 23: 883-889.

18. Roaiah MF, El Khayat YI, GamalEl Din SF, Abd El Salam MA (2015) Pilot study on the effect of botanical medicine (Tribulus terrestris) on serum testosterone level and erectile function in aging males with partial androgen deficiency (Padam). J Sex Marital Ther 7: 1-5.

19. Moeloek N, Adimoelja A, Tanojo T, Pangkahila W (1994) Trilas of Tribulus terrestris on oligozoospermia. Proceedings of the VI National Congress and III International Symposium on New Perspectives on Andrology in Human Reproduction. National Congress of Indonesian Association of Andrology (PANDI).

20. Antonio L, Uelmen J, Rodruiguez R, Earnest C (2000) The effects of Tribulus terrestris on body composition and exercise performance in resistane-trained males. Int J Sport Nutr Exerc Metab 10: 208-215.

21. Marco Pautasso (2013) Ten simple rules for writing a literature review. PLoS Comput Biology 9: e1003149.

22. Rogerson S, Riches CJ, Jennings C, Weatherby RP, Meir RA, et al. (2007) The effect of five weeks of Tribulus terrestris supllementation on muscle strength and body composition during preseason training in elite rugby league players. J Strengh Cond Res 21: 348-353.

23. https://www.wada-ama.org/en/media/news/2016-09/wadapublishes-2017-prohibited-list

24. Ma Y, Guo Z, Wang X (2015) Tribulus terrestris extracts alleviate muscle damage and promote anaerobic performance of trained male boxers and its mechanisms: Roles of androgen, IGF-1, and IGF binding protein-3. J Sport Health Sci.

25. Vandewalle H, Pérès G, Monod H (1987) Standard anaerobic exercise tests. Sports Med 4: 268-289.

26. J. Frystyk (2010) Exercise and the growth hormone-insulin-like growth factor axis. Med Sci Sports Exerc 42: 58-66.

27. Adimoelja A, Adaikan PG (1997) Protodioscin from herbal plant Tribulus terrestris $\mathrm{L}$. improves the male sexual functions, probably via DHEA. Int J Impotence Res 9: 1-15. 